\title{
CHALLENGES IN HADRON PHYSICS
}

\author{
Ulf-G. Meißner \\ Helmholtz-Institut für Strahlen- und Kernphysik (Theorie), Universität Bonn \\ Nußallee 14-16, D-53115 Bonn, Germany \\ and \\ Institut für Kernphysik (Theorie), Forschungszentrum Jülich \\ D-52425 Jülich, Germany \\ Received (Day Month Year) \\ Revised (Day Month Year)
}

In this talk, I address some open problems in hadron physics and stress their importance for a better understanding of QCD in the confinement regime.

Keywords: Hadrons; pentaquarks; strangeness

\section{Introductory remarks}

Hadron physics explores the least understood sector of the Standard Model (SM), namely QuantumChromoDynamics (QCD) at large gauge coupling. In the region of energies below a few $\mathrm{GeV}$, one is faced with two different types of challenges:

(i) Precision calculations/measurements: Many very precise data exist which let one extract fundamental QCD parameters (condensates, quark masses, etc.) or explore bounds for physics beyond the SM. In such cases, precision calculations to accuracies of a few percent or better have to be done, which in certain cases can be achieved utilizing effective field theory techniques. However, it should be stressed that contrary to popular opinion, more such precise data are needed, e.g. in processes involving strange quarks. Space does not allow to discuss these very interesting developments here.

(ii) The spectrum: To really understand the issue of confinement in the light quark sector, we must first bring order into the spectrum. The nature and the spectrum of hadrons can only be understood if we are able to say with certainty which states are genuine quark model states, which are dynamically generated through channel couplings or which ones are exotic. For that, precise measurements of many properties of these states are needed and theoretical tools have to be developed to separate the often overlapping resonances. In this talk, I will address two topics related to this type of problems, which are a) some properties of the exotic $\Theta^{+}(1540)$ (the so-called pentaquark) and b) the way to achieve a 
unified description of resonances. Clearly, this choice is very subjective, so many other interesting developments and/or challenges could not be discussed.

\section{Pentaquark issues I: Determining its parity}

The parity of the recently discovered exotic state $\Theta^{+}(1540)$ with positive strangeness is not yet determined ${ }^{1}$ (I refer to the various plenary talks given at this conference for a detailed discussion on the various experiments and possible criticism as well as on theoretical approaches). Its parity is considered a decisive quantity regarding its substructure. The most appealing proposal to determine the parity is based on the application of the Pauli principle in the process $\vec{p} \vec{p} \rightarrow \Theta^{+} \Sigma^{+}[2$ since it links spin and parity. In 3 the spin correlation coefficient $A_{x x}$ was identified as the crucial observable since in the low-energy region its sign is directly related to parity of the $\Theta^{+}$, see Fig. 1 and also Table 1 (assuming that the $\Theta^{+}$has spin $1 / 2$ ). Due to the self-analyzing hyperon decay, the energy dependence of spin transfer coefficient $D_{x x}$ might also be used to determine $\pi\left(\Theta^{+}\right)$(under certain favorable conditions), cf. again Table 1 (where the threshold values/ranges are given). In these arguments, one assumes naturalness of the contributing partial waves, in particular for the S-waves that dominate the region of small excess energy $Q$.

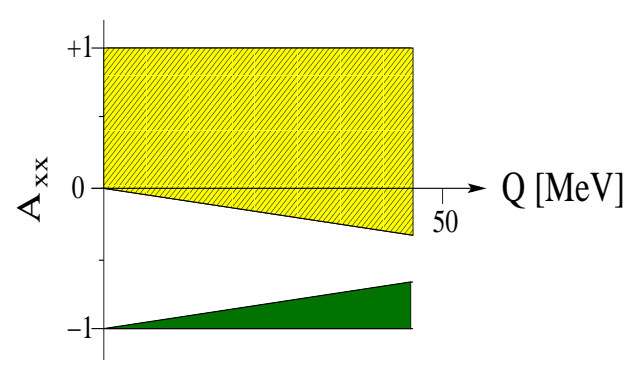

Fig. 1. Schematic presentation of the result for $A_{x x}$ for the two possible parity states of the $\Theta^{+}$. For either option realized the corresponding data should fall into the area indicated. In case of a negative parity the threshold value depends on the ratio of the strength of the two possible $\mathrm{S}-$ wave amplitudes.

Table 1. Spin transfer and spin correlation coefficients for positive/negative $\Theta^{+}$parity in $\vec{p} \vec{p} \rightarrow \Theta^{+} \Sigma^{+}$at threshold. For definitions, see e.g. Ref. 4 .

\begin{tabular}{|cc|cc|cc|cc|}
\hline$D_{x x}(+)$ & $D_{x x}(-)$ & $D_{z z}(+)$ & $D_{z z}(-)$ & $A_{x x}(+)$ & $A_{x x}(-)$ & $A_{z z}(+)$ & $A_{z z}(-)$ \\
\hline 0 & {$[-1 / \sqrt{2}, 1 / \sqrt{2}]$} & 0 & {$[0,1]$} & -1 & {$[-1,1]$} & -1 & {$[0,1]$} \\
\hline
\end{tabular}

In 5 are more detailed study of these proposals was performed, in particular, models were constructed were the leading S-waves are suppressed due to cancellations, thus explicitely violating the naturalness assumption. Still, it was shown that the energy dependence of ${ }^{3} \sigma_{\Sigma}=\sigma_{0}\left(1+\left(A_{x x}+A_{y y}\right) / 2\right)$, with $\sigma_{0}$ the unpolarized cross section, guarantees unambiguous information on the parity of the $\Theta^{+}$. This can be achieved e.g. by measurements at two $Q$-values, say at 20 and $40 \mathrm{MeV}$, to avoid the Coulomb effects at smaller excess energies but still staying sufficiently close to threshold. For a more detailed discussion (in particular the the usefulness of the quantity $\sigma_{0} D_{x x}$ ) and more references on this issue, please consult 5 . 


\section{Pentaquark issues II: The width of the $\Theta^{+}$}

Another interesting property of the $\Theta^{+}$is its width. Most experiments which have seen evidence of this state only quote upper limits for the width given by the resolution of the experiment. For example, the fairly clean signal in proton-proton collisions reported by the COSY-TOF collaboration $\left[6\right.$ gives $\Gamma_{\Theta^{+}} \leq(18 \pm 4) \mathrm{MeV}$ due to the energy resolution. In various papers, information on kaon-nucleon scattering ${ }^{7}$ was analyzed leading to the conclusion that the width of the $\Theta^{+}$has to be less than a few MeV. A strongly interacting particle with such a small width could truly be called "exotic". In $[8$ the impact of the pentaquark on differential and integrated cross sections for the reaction $K^{+} d \rightarrow K^{0} p p$, where experimental information is available at kaon momenta below $640 \mathrm{MeV}$ (the mass range of 1520 to $1555 \mathrm{MeV}$ corresponds to kaon momenta in the range $417 \leq k_{0} \leq 476 \mathrm{MeV}$ ), was investigated. The calculation utilizes the Jülich kaon-nucleon model (see 9 and references therein) and an extension of it that includes the contribution of a $\Theta^{+}(1540)$ with a variable width. The evaluation of the reaction $K^{+} d \rightarrow K^{0} p p$ takes into account effects due to the Fermi motion of the nucleons within the deuteron and three-body kinematics.

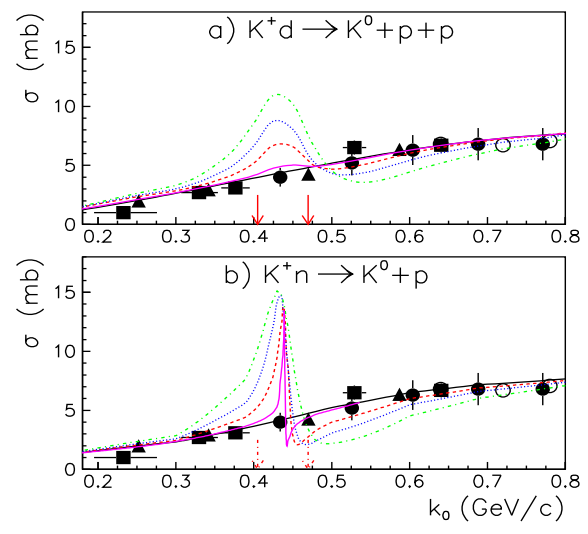

Fig. 2. Total $K^{+} d \rightarrow K^{0} p p$ cross section as a function of the kaon momentum. The lines in a) show our results for the $K^{+} d \rightarrow K^{0} p p$ reaction obtained with different $\Theta^{+}$widths: $\Gamma_{\Theta}=1 \mathrm{MeV}$ - solid, $5 \mathrm{MeV}$-dashed, $10 \mathrm{MeV}$-dotted and $20 \mathrm{MeV}$-dashed-dotted, while the solid (black) line is our calculation without pentaquark. The lines in b) show the calculations for the $K^{+} n \rightarrow K^{0} p$ reaction assuming that the neutron target is at rest.

As shown in Fig. 2, one concludes from that analysis that the data constrain the width of the $\Theta^{+}(1540)$ to be less than $1 \mathrm{MeV}$. In 10 , the reaction $K^{+} X e \rightarrow K^{0} p X$ was investigated in a meson-exchange model including rescattering of the secondary protons with the aim to analyze the evidence for the $\Theta^{+}(1540)$ reported by the DIANA collaboration 11. It was confirmed that the kinematical cuts introduced by the DIANA collaboration efficiently suppress the background to the $K^{+} n \rightarrow K^{0} p$ reaction which may contribute to the $\Theta^{+}$production. These kinematical cuts do not produce a narrow structure in the $K^{0} p$ effective mass spectrum near $1540 \mathrm{MeV}$, cf. the left panel of Fig. 3. The effect of a narrow resonance with both positive and negative parity in comparison to the DIANA data was studied in 10 . It is shown that the $K^{+} X e \rightarrow K^{0} p X$ calculations without $\Theta^{+}$contribution as well as the results obtained with a $\Theta^{+}$width of $1 \mathrm{MeV}$ are in comparably good agreement with the DIANA results, see Fig. 3. The $\chi^{2} /$ dof is $2.3,2.7$ and 2.9 for no $\Theta^{+}$, a positive 
and a negative parity spin- $1 / 2$ pentaquark with a width of $1 \mathrm{MeV}$, in order (with the cuts applied to the $K^{0} p$ invariant mass spectrum). In view of these results, the three star rating for the $\Theta^{+}$in the recent PDG tables seems somewhat optimistic 1. More dedicated experiments are called for to establish (or rule out) this exotic baryon resonance.
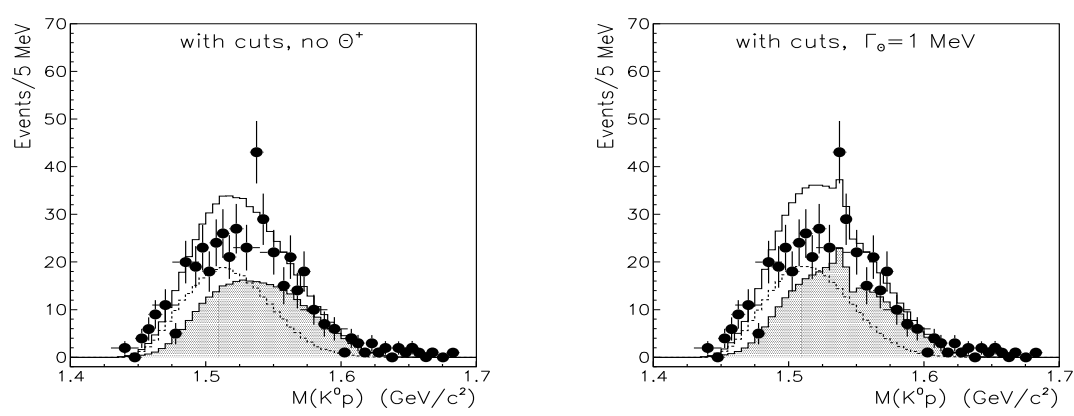

Fig. 3. The $K^{0} p$ invariant mass spectrum from the $K^{+} X e$ reaction with the kinematical cuts performed by the DIANA collaboration. The solid histograms are the results without (left)/with the inclusion of a $\Theta^{+}$with a width of $1 \mathrm{MeV}$ (right). Hatched histograms show the spectrum from the direct $K^{+} n \rightarrow K^{0} p$ production on a bound neutron, for the dashed curve an additional single rescattering of the proton was taken into account, while the solid curve is their sum.

\section{Unified description of resonances}

This section first presents some results on the baryon spectrum (for definiteness, a few states are selected to make the point) using two very different approaches. In the second more speculative part some ideas are presented how one could unify these approaches and eventually gain a deeper insight into the confinement regime of QCD.

\subsection{Generating hyperon resonances}

The quark model has been particularly successful in bringing order into the many observed meson and baryon resonances. In the last years, it has become evident that a covariant framework is necessary to deal with all the (ir)regularities found in the hadron spectrum. Here, I will focus on the baryon resonances and pick one particular covariant quark model, in which the residual interaction of the constituent quarks are a flavor-dependent instanton induced interaction and a linear confinement potential (for details, see 12 ). This approach leads to a fairly good description of the spectrum, including states with very high spin and is consistent with Regge phenomenology. The 't Hooft interaction also explains the near degeneracy of many positive and negative parity states 13 , which is very different from the speculations 
about chiral restauration in the hadron spectrum at masses above $\simeq 1.8 \mathrm{GeV}$ which one finds frequently in the current literature. Many electroweak observables 14 and heavy quark properties have also been successfully calculated. Strong pion decays come out typically a factor two too small, but the overall pattern of pion couplings is well described since 15 the so-called "missing resonances" have couplings that are at least an order of magnitude smaller than for the states that couple with normal strength to the pion-nucleon continuum. Just to be precise, in this approach the $\Lambda(1405)$, the $\Sigma(1620)$ and the $\Lambda(1670)$ are three-quark states 16 . Note, however, that the $\Lambda(1405)$ is not well described in this approach, pointing towards the boundstate scenario discussed below. Furthermore, in the framework of this model, one also generates a whole zoo of states with quark content $q q q q \bar{q}$, some of them exotic and many of them with conventional quantum numbers, which also has to be kept in mind in the discussion of the pentaquark $\Theta^{+}$- there simply can not just be one state like that, there must be many (so far one experiment has a hint for another exotic baryon from the anti-decuplet 17 ). [For a different modern quark model, see e.g. 18 .

Next, I discuss how hyperon resonances can be generated as meson-baryon bound states. For that, consider $K^{-} p$ scattering. A purely perturbative treatment is not possible due to the strong channel couplings and the appearance of a subthreshold resonance, the $\Lambda(1405)$. A non-perturbative resummation scheme is mandatory to generate a bound state or a resonance. There exist many such approaches, but it is possible and mandatory to link such a scheme tightly to the chiral QCD dynamics. Such an improved approach was developed for pion-nucleon 19 and later applied to $\bar{K} \mathrm{~N}$ scattering 20 . The starting point is the T-matrix for any partial wave, which can be represented in closed form if one neglects for the moment the crossed channel (left-hand) cuts, $T=1 /\left[\tilde{T}^{-1}(W)+g(s)\right]$, with $W=\sqrt{s}$ the cm energy. $\tilde{T}$ collects all local terms and poles and $g(s)$ is the meson-baryon loop function (the fundamental bubble) that is resummed by e.g. dispersion relations in a way to exactly recover the right-hand (unitarity) cut contributions. The function $g(s)$ needs regularization, this can be best done in terms of a subtracted dispersion relation and using dimensional regularization. It is important to ensure that in the low-energy region, the so constructed T-matrix agrees with the one of CHPT (matching). In addition, one has to recover the contributions from the left-hand cut. This can be achieved by a hierarchy of matching conditions, which enforce that to a given order in the chiral expansion, the unitarized amplitude agrees with the CHPT amplitude (eventually in some unphysical region). Such a procedure tightly constrains the unitarization procedure, for details see 19. It was observed in 20 that there are indeed two poles close to the nominal $\Lambda(1405)$ resonance. The physics behind these two poles was recently revealed in 21. Starting from an $\mathrm{SU}(3)$ symmetric Lagrangian to couple the meson octet to the baryon octet (in that limit, all octet Goldstone boson masses and all octet baryon masses are equal), one could in principle generate a variety of resonances according to the 
SU(3) decomposition, $8 \otimes 8=1 \oplus 8_{s} \oplus 8_{a} \oplus 10 \oplus \overline{10} \oplus 27$. As it turns out, the leading order transition potential is attractive only in the singlet and the two octet channels, so that one a priori expects a singlet and two octets of bound states. However, the two octets come out degenerate. This has no particular dynamical origin but rather is a consequence of the actual values of the $\mathrm{SU}(3)$ structure constants. In the real world, there is of course $\mathrm{SU}(3)$ breaking of various origins. This was parameterized in 21 in terms of a symmetry breaking parameter $x$ in the expressions for the meson $M_{i}$ and baryon masses $m_{i}$ as well as the subtraction constants $a_{i}$ via $M_{i}^{2}(x)=$ $M_{0}^{2}+x\left(M_{i}^{2}-M_{0}^{2}\right), m_{i}(x)=m_{0}+x\left(m_{i}-m_{0}\right)$ and $a_{i}(x)=a_{0}+x\left(a_{i}-a_{0}\right)$, with $M_{0}=368 \mathrm{MeV}, m_{0}=1151 \mathrm{MeV}$ and $a_{0}=-2.148$, where $0 \leq x \leq 1$. The motion of the various poles in the complex energy plane as a function of $x$ is shown in Fig. 4. We note that the two octets split, in particular, one moves to lower energy $(I=0,1426 \mathrm{MeV})$ close to the position of the singlet $(I=0,1390 \mathrm{MeV})$. These are the two poles which combine to give the $\Lambda(1405)$ as it appears in various reactions. Recent kaon photoproduction data from Spring- 8 seem to give support to this twopole scenario. We note that the $\Sigma(1620)$ and the $\Lambda(1670)$ are also generated (the latter is the right-most pole in Fig. 4), besides other resonances with strangeness $S=-1$, see Ref. $\frac{2223}{2}$ and also the extensive studies in 24 .

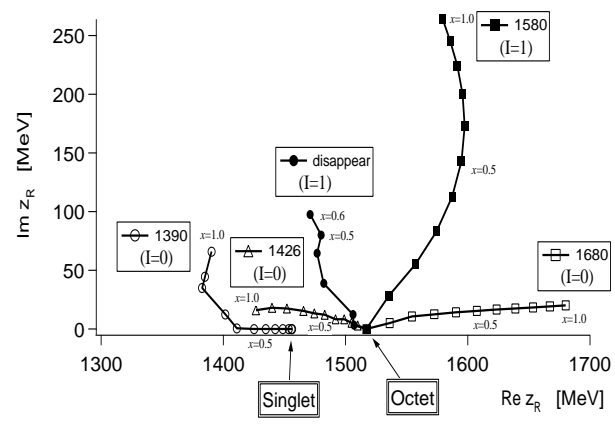

Fig. 4. Trajectories of the poles in the scattering amplitudes obtained by changing the $\mathrm{SU}(3)$ breaking parameter $x$ gradually. In the $\mathrm{SU}(3)$ limit $(x=0)$, only two poles appear, one corresponding to the singlet and the other to the two degenerate octets. The symbols correspond to the step size $\delta x=0.1$.

\subsection{Combining the quark model and coupled channel dynamics}

Here, I will speculate how one can bring together the quark model and the coupled channel approach to achieve a unified description of resonances. For that, one can follow a two-step procedure, as discussed below. Before going into the details, I remark that step 1 has been done (to some extent) whereas the second step so far is science fiction, but I consider it an important topic to be addressed in the coming years. More precisely, I envision the following:

Step 1: The coupled channel approach can be extended in that one can also include resonance fields by saturating the local contact terms in the effective Lagrangian through explicit meson and baryon resonances (for details, see ${ }^{19}$ ). In particular, in this framework one can cleanly separate genuine quark resonances from dynamically generated resonance-like states. The former require the inclusion of an explicit field in the underlying Lagrangian, whereas in the latter case the fit will arrange itself so 
that the couplings to such an explicit field will vanish. This framework was applied in 19 to the case of pion-nucleon scattering below the $\eta N$ threshold. Some typical results are: 1) The delta pole is located at $(1210, i 53) \mathrm{MeV}$, in agreement with dispersion-theoretical studies of pion-nucleon scattering and pion photoproduction. Furthermore, a genuine 3-quark component is needed to build up this state. 2) The rho requires a quark-antiquark component, consistent with large $\mathrm{N}_{C}$ investigations and its tensor coupling comes out large, $\kappa_{\rho} \simeq 6.3$. 3) No light scalar is needed to describe the data, all the interaction in the t-channel with scalar-isoscalar quantum numbers is build up by pion rescattering (loops). This is consistent with the analysis of other reactions, like e.g. $J / \Psi \rightarrow V \pi \pi / \bar{K} K$ (with $V=\omega, \phi){ }^{[25]}$ or the scalar form factor of the pion 26 as well as the intermediate range attraction in the central part of the two-nucleon potential.

Step 2: A further step would be to merge the coupled channel dynamics with quark model predictions. In Fig. 5 the underlying idea is sketched for the example of the strong $\Delta \rightarrow N \pi$ transition. In a pure quark model description based e.g. on the Bethe-Salpeter formalism, one can only describe part of the $\Delta \rightarrow N \pi$ decay, since there is a sizeable modification of the vertex through final state interactions (often called "dressing") and also channel coupling (some typical graphs are shown on the right-hand-side of that figure). In fact, the idea would be to use the vertices and masses generated in the quark model as input in these loop corrections. That way, the aforementioned problem of the too small strong width should be solved and one also obtains a better representation of the underlying dynamics. Ultimately, this should be extended to many channels so as to achieve a truly unified description of the spectrum of the strongly interacting particles and their properties. To come back to the hyperon states discussed before, such a scheme would allow one to really pin down the nature of these states, my guess is that while the $\Lambda(1670)$ and the $\Sigma(1620)$ are dominantly three-quark states, the $\Lambda(1405)$ will not have a three-quark component but simply be a dynamically generated state with different response to external probes and decay patterns. To end this section, I should point out that such ideas are not entirely new, but in my opinion only now the theoretical tools have become available to seriously tackle this problem.

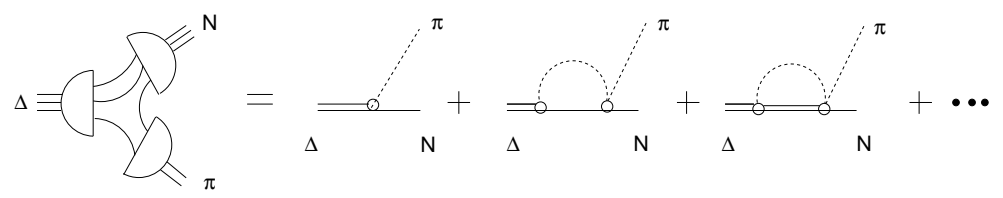

Fig. 5. A schematic representation of merging the quark model with the coupled channel dynamics. The quark model can be used to generate the bare particle masses and vertices, which are renormalized in a consistent fashion (unitarized CHPT) as shown by some typical one-loop graphs. Higher loop graphs that are not shown here are also generated by the unitarization procedure. 


\section{Concluding remarks}

I would like to stress again that hadron structure and dynamics is one of the central issues of QCD (of the Standard Model) and require non-perturbative methods in the few $\mathrm{GeV}$ region and below. Apart from the topics discussed before, there are many further challenges related to (I just name a few):

- Lattice QCD and chiral extrapolations. Most lattice data are obtained for large unphysical values of the quark (pion) masses and thus must be interpolated by means of chiral perturbation theory to the physical value of $M_{\pi}$. On the example of the nucleon mass, the possible loopholes and limitations of such methods are discussed in 27. It is evident that for pion masses larger than $500 \mathrm{MeV}$, chiral extrapolations cease to be useful.

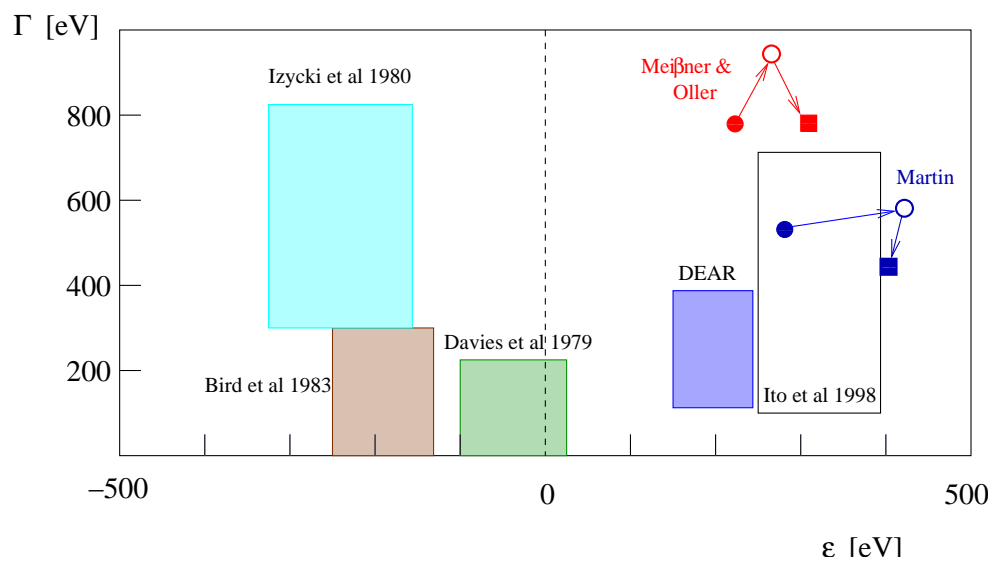

Fig. 6. Predictions for the ground-state strong shift $\Delta E_{1}^{s}$ and the width $\Gamma_{1}$. Filled circles correspond to using the Deser formula, empty circles using the scattering amplitude instead of the scattering length (thus including the large cusp correction due ot the $\bar{K}^{0} n$ channel) and filled boxes the final formula derived in 31 .

- As noted in the introduction, precision hadronic calculations are needed to e.g. really be able to address the issue whether physics beyond the SM has been seen in the precise BNL measurements of the muon anomalous magnetic moment 28 (my guess would be not) or if one wants to precisely test CKM unitarity in B-decays. Another recent example are the radiative corrections to tritium $\beta$-decay $[29]$ to reduce the upper limit on the (electron) neutrino mass 30 . In addition, there are some real puzzles when comparing precision data to precision calculations. As an example, in Fig. 6 a calculation of the ground-state energy shift and width of kaonic hydrogen 31 compared to earlier measurements 32 and the recent result from DEAR 33 is shown. As the only input, the S-wave scattering length combination $\left(a_{0}+a_{1}\right) / 2$ was taken from the dispersive analysis of Martin 34 and from the unitarized CHPT analysis of 20]. In both cases, the 
theoretical prediction (filled boxes) is in stark disagreement with the DEAR result. At present, this discrepancy is unexplained.

- There has been impressive progress made in Nuclear Effective Field Theory. In particular, two- and three-nucleon forces can be described in one consistent scheme. By now, the nucleon-nucleon force has been worked out to next-tonext-to-next-to-leading order in the EFT expansion $[35] 36$ and has reached the accuracy needed to do precise calculations in few-nucleon systems. Other advances in this field concern the quark mass dependence of light nuclei 3738 and the speculation of an infrared renormalization group limit cycle in QCD 39 . For many other applications, in particular low energy reactions of relevance for astrophysics and element synthesis, see e.g. Refs! 4041 .

\section{Acknowledgments}

I would like to thank the organizers to give me this opportunity, my collaborators J. Haidenbauer, C. Hanhart, D. Jido, J.A. Oller, E. Oset, U. Raha, A. Ramos, A. Rusetsky and A. Sibirtsev as well as B.C. Metsch and H.R. Petry for sharing their insight into the topics discussed here. I have also profited from collaborations with V. Bernard, E. Epelbaum, S. Gardner, H.-W. Hammer and W. Glöckle. Special thanks to Paulo Bedaque and the Nuclear Theory Group at LBNL for their hospitality where part of this work was done.

\section{References}

1. S. Eidelman et al., Phys. Lett. B 592 (2004) 1.

2. A. W. Thomas, K. Hicks and A. Hosaka, Prog. Theor. Phys. 111 (2004) 291 arXiv:hep-ph/0312083.

3. C. Hanhart et al., Phys. Lett. B 590 (2004) 39 arXiv:hep-ph/0312236.

4. C. Hanhart, Phys. Rept. 397 (2004) 155 arXiv:hep-ph/0311341.

5. C. Hanhart, J. Haidenbauer, K. Nakayama and U.-G. Meißner, arXiv:hep-ph/0407107

6. M. Abdel-Bary et al. [COSY-TOF Collaboration], arXiv:hep-ex/0403011

7. S. Nussinov, arXiv:hep-ph/0307357 R. N. Cahn and G. H. Trilling, Phys. Rev. D 69 (2004) 011501 arXiv:hep-ph/0311245; R. A. Arndt, I. I. Strakovsky and R. L. Workman, Phys. Rev. C 68 (2003) 042201 [Erratum-ibid. C 69 (2004) 019901] arXiv:nucl-th/0308012; J. Haidenbauer and G. Krein, Phys. Rev. C 68 (2003) 052201 arXiv:hep-ph/0309243; W. R. Gibbs, arXiv:nucl-th/0405024

8. A. Sibirtsev, J. Haidenbauer, S. Krewald and U.-G. Meißner, arXiv:hep-ph/0405099

9. M. Hoffmann, J. W. Durso, K. Holinde, B. C. Pearce and J. Speth, Nucl. Phys. A 593 (1995) 341 arXiv:nucl-th/9504029.

10. A. Sibirtsev, J. Haidenbauer, S. Krewald and U.-G. Meißner, arXiv:nucl-th/0407011

11. V. V. Barmin et al. [DIANA Collaboration], Phys. Atom. Nucl. 66 (2003) 1715 [Yad. Fiz. 66 (2003) 1763] arXiv:hep-ex/0304040.

12. U. Löring, K. Kretzschmar, B. C. Metsch and H. R. Petry, Eur. Phys. J. A 10 (2001) 309 arXiv:hep-ph/0103287.

13. U. Löring and B. Metsch, arXiv:hep-ph/0110412

14. D. Merten, U. Löring, B. Metsch and H. Petry, Eur. Phys. J. A 18 (2003) 193.

15. B. Metsch, U. Löring, D. Merten and H. Petry, Eur. Phys. J. A 18 (2003) 189. 
16. U. Löring, B. C. Metsch and H. R. Petry, Eur. Phys. J. A 10 (2001) 447 arXiv:hep-ph/0103290.

17. C. Alt et al. [NA49 Collaboration], Phys. Rev. Lett. 92 (2004) 042003 arXiv:hep-ex/0310014.

18. L. Y. Glozman, W. Plessas, K. Varga and R. F. Wagenbrunn, Phys. Rev. D 58 (1998) 094030 arXiv:hep-ph/9706507; R. F. Wagenbrunn, S. Boffi, W. Klink, W. Plessas and M. Radici, Phys. Lett. B 511 (2001) 33 arXiv:nucl-th/0010048; S. Boffi, L. Y. Glozman, W. Klink, W. Plessas, M. Radici and R. F. Wagenbrunn, Eur. Phys. J. A 14 (2002) 17 arXiv:hep-ph/0108271.

19. U.-G. Meißner and J. A. Oller, Nucl. Phys. A 673 (2000) 311 arXiv:nucl-th/9912026 .

20. J. A. Oller and U.-G. Meißner, Phys. Lett. B 500 (2001) 263 arXiv:hep-ph/0011146.

21. D. Jido, J. A. Oller, E. Oset, A. Ramos and U.-G. Meißner, Nucl. Phys. A 725 (2003) 181 arXiv:nucl-th/0303062.

22. E. Oset, A. Ramos and C. Bennhold, Phys. Lett. B 527 (2002) 99 [Erratum-ibid. B 530 (2002) 260] arXiv:nucl-th/0109006.

23. S. Sarkar, E. Oset and M. J. V. Vacas, arXiv:nucl-th/0407025

24. E. E. Kolomeitsev and M. F. M. Lutz, Phys. Lett. B 585 (2004) 243 arXiv:nucl-th/0305101; M. F. M. Lutz and E. E. Kolomeitsev, Nucl. Phys. A 730 (2004) 110 arXiv:hep-ph/0307233; arXiv:nucl-th/0402084

25. U.-G. Meißner and J. A. Oller, Nucl. Phys. A 679 (2001) 671 arXiv:hep-ph/0005253.

26. U.-G. Meißner, Comments Nucl. Part. Phys. 20 (1991) 119.

27. V. Bernard, T. R. Hemmert and U.-G. Meißner, Nucl. Phys. A 732 (2004) 149 arXiv:hep-ph/0307115.

28. G. W. Bennett et al. [Muon g-2 Collaboration], Phys. Rev. Lett. 92 (2004) 161802 arXiv:hep-ex/0401008.

29. S. Gardner, V. Bernard and U.-G. Meißner, arXiv:hep-ph/0407077

30. A. Osipowicz et al. [KATRIN Collaboration], arXiv:hep-ex/0109033

31. U.-G. Meißner, U. Raha and A. Rusetsky, Eur. Phys. J. C 35 (2004) 349 arXiv:hep-ph/0402261.

32. J. D. Davies et al., Phys. Lett. B 83 (1979) 55; M. Izycki et al., Z. Phys. A 297 (1980) 11; P. M. Bird et al., Nucl. Phys. A 404 (1983) 482; M. Iwasaki et al., Phys. Rev. Lett. 78 (1997) 3067.

33. G. Beer et al. [DEAR Collaboration], Prog. Theor. Phys. Suppl. 149 (2003) 240.

34. A. D. Martin, Nucl. Phys. B 179 (1981) 33.

35. D. R. Entem and R. Machleidt, Phys. Rev. C 68 (2003) 041001 arXiv:nucl-th/0304018.

36. E. Epelbaum, W. Glöckle and U.-G. Meißner, arXiv:nucl-th/0405048

37. S. R. Beane and M. J. Savage, Nucl. Phys. A 713 (2003) 148 arXiv:hep-ph/0206113.

38. E. Epelbaum, U.-G. Meißner and W. Glöckle, Nucl. Phys. A 714 (2003) 535 arXiv:nucl-th/0207089.

39. E. Braaten and H. W. Hammer, Phys. Rev. Lett. $91 \quad$ (2003) 102002 arXiv:nucl-th/0303038.

40. S. R. Beane, P. F. Bedaque, W. C. Haxton, D. R. Phillips and M. J. Savage, in M. Shifman (ed.): At the frontier of particle physics, Vol. 1., pp. 133-269, arXiv:nucl-th/0008064.

41. T. S. Park, K. Kubodera, D. P. Min and M. Rho, Nucl. Phys. A 684 (2001) 101 arXiv:nucl-th/0005069. 